\title{
The role of baseline granularity for benchmarking citation impact. The case of CSS profiles
}

\author{
Wolfgang Glänzel ${ }^{1,2}$ (D) Bart Thijs ${ }^{3}$
}

Received: 15 January 2018/Published online: 27 April 2018

(C) Akadémiai Kiadó, Budapest, Hungary 2018

\begin{abstract}
In this paper we study the effect of granularity on Characteristic Scores and Scales (CSS). Unlike the traditional indicators that are mostly based on means and quantiles, CSS require the reduction of the citation distributions collaboration of the underlying reference population to four states (classes) and thus higher a different level of granularity. While the question of the choice of granularity is at higher levels of aggregation usually not critical since countries and university have rather multidisciplinary profiles, at lower aggregation levels specialisation becomes more typical. Inappropriate granularity might not warrant the depiction of the publication profiles at these levels in a correct and adequate manner and thus not add accurate citation profiles either. In order to be able to process one complete annual volume of the Web of Science, we decided to calculate CSS thresholds and classes for two levels of granularity, namely sub-fields and WoS Subject Categories. With about 5\% deviation, we did not find a real significance. However, we identified journals with similar impact measures but different citation profiles, independently of the granularity. Finally, we have pointed to the limitations in the choice of granularity - in terms of both too broad and too narrow subjects.
\end{abstract}

Keywords Granularity - Citation impact - Characteristic scores and scales · Journal citation measures

Wolfgang Glänzel

wolfgang.glanzel@kuleuven.be; glanzw@iif.hu

Bart Thijs

bart.thijs@kuleuven.be

1 ECOOM and Dept MSI, KU Leuven, Louvain, Belgium

2 Dept Science Policy and Scientometrics, Library of the Hungarian Academy of Sciences, Budapest, Hungary

3 ECOOM, KU Leuven, Louvain, Belgium 


\section{Introduction}

The question of granularity in the application of subject-based standards remains a constantly recurring issue. The gain of higher resolution and precision of details at zooming on the hierarchy of subject assignment is lost again by growing ambiguity and increasing fuzziness. Apart from randomness, Bookstein (1997) has identified two other demons being challenges to the measurement in informetrics and quantitative science studies. He pointed to fuzziness as the second demon reaching beyond probabilistic uncertainty as it is related to the impossibility to accurately represent concepts by traditional variable sets. Finally, ambiguity pushes the idea of fuzziness to its extreme: The conceptual basis for measurement itself is often weak although we aim at accurate measurement. These demons do also affect the possibility of accurate subject assignment in bibliometric applications as the conceptual basis underlying subject delineation and classification is often weak. In particular, we can identify two main weak points regarding subject classification. The first one concerns the way how subject assignment is made and the second one relates to the granularity of subject hierarchies. The need for accuracy is often confronted with the practical limitations as well. These limitations may results from indirect assignment (e.g., through journal classification), hard clustering or even by changes in context and perspective, that is, by the question of research in a special topic is still relevant for the subject in question or for the activity of a department under study. While at higher level of aggregation this effect may still be tolerable and benchmarking may still lead to acceptable results if high-level subject classification is used, for instance, in national comparison of publication activity and citation impact in fields like chemistry or biosciences, at lower levels of aggregation, at least at the level of scientific journals, university departments and research teams this approach is stretched to its limits as the profiles of these units are very specific and require more individual approaches. Also different publication types might require different levels of granularity as Adams and Testa (2011) stressed in the context of the-at that time-newly introduced Book Citation Index (Thomson Reuters, now Clarivate Analytics-sBKCI). At lower aggregation levels also first dramatic conflicts between subject delineation and classification become blatantly obvious. In other words, acceptable delineation is often not possible by using standard classification alone. Ideally quasiunique paper-based assignment of high resolution would be a solution but in practice this might turn counterproductive as further zooming in may result in highly grainy blow-ups that may destroy the details, thus shapes and contours become fuzzy. The desire to correct ambiguity and fuzziness and to sharpen the blurred picture by supplementary but arbitrary human decisions and selections resembles a bit the allegory of reality, dream and interpretation shown using the example of the photographer's interaction with material and technology in Michelangelo Antonioni's film "Blow-Up" (1966), where the attempt to blow up a photograph to gain more information and lucidity and to get evidence finally resulted in the destruction of the detail structure just leaving even more room for obscurity, imagination and speculation (cf. Lehmann 2013).

Coming back to bibliometrics, we will study the effect in borderline applications. We have used two levels of granularity for achieving this objective, the level of scientific journals and the Web of Science Subject Categories. 


\section{General methodological issues}

The question of granularity has been raised and analysed in recent studies. Among those studies we would like to bring out three articles that have been devoted to the analysis of the stability of relative indicators in the light of granularity. The authors of the first one (Zitt et al. 2005) observed a certain "instability of impact measures should not be interpreted in terms of lack of robustness but rather as the coexistence of various perspectives each having their own form of legitimacy". In default of 'one best level' of observation they therefore pled for particular prudence in interpretation of citation indicators instead of just choosing or preferring one of the possible worlds. Adams et al. (2008) have tested the hypotheses stated by Zitt et al. using the example of the 2001 UK Research Assessment Exercise (RAE) and found that the correlation between average normalised citation impact and peer-reviewed grade varied according to the hierarchical selected level. Both studies use journals and different hierarchical levels of subject classification. When interpreting the results, we have to take two important issues into account. The first one is simply a methodological one; journals form a partition, while subject classification do not. Subjects always overlap to a certain extent, where the extent depends on the hierarchical level and, of course, on the underlying scheme chosen for the exercise as well. In order to overcome the usual challenge of overlapping subject categories and disciplines, Zitt et al., have forced single subject assignment of journals to subject categories and of subject categories to disciplines thus reaching partitioning the document space in all cases. Although this seems to be quite reasonable and convincing at the first sight, we have to point to the following crucial issue. The second issue is much more critical as it is of conceptual nature. Although in the Clarivate Analytics Web of Science Core collection (Wos) and the Scopus databases subject fields are defined on the basis of journal assignment, there is a fundamental difference between journal- and subject-based standards. Authors are bound up with their specialty due to education, their skills and career choices, but the discipline is not individually selected for each publication. Yet in the case of journals authors do decide where to publish their manuscript. Thus journals always reflect certain aspects of publication strategies since the authors have the-admittedly sometimes limited-freedom to choose the journal standard along with the scope for the target audience. In order to illustrate this, we refer to our plain old indicator triplet consisting of the mean observed, (journal) expected and field expected citation rates (MOCR, MECR and FECR, cf. Glänzel et al. 2009). MECR and FECR provide two different expectations and the comparison of these two baselines reveal interesting details about the analysed unit's publication profiles and strategies. For instance, MOCR $>$ MECR $>$ FECR means that the factual citation impact is above both expectations, where scientists affiliated with this unit, on an average, publish in journals with citation impact higher that the field standard, while MECR > MOCR > FECR means that the unit exhibits a factual citation impact above the expected subject-based standard and it does publish in high-impact journals but this standard is, on an average, not reached. What remains, is the comparison of granularity effects at different hierarchical level indeed. In 2009 we have made therefore an experiment using the example of 676 European universities with at least 50 publications each in the period 1999-2001 that have been selected from a total of roughly 2000 institutions (see Glänzel et al. 2009).

The 3-year citation-window based field-expected citation rate (FECR) was determined on the basis of different hierarchical levels of subject classifications, the WoS subject categories, the 60 sub-fields and 15 major field according to the Leuven-Budapest subject 
classification scheme, which is built upon the WoS categories (cf. Glänzel and Schubert, 2003). Two of the results are shown in Fig. 1. NMCR stands for the ratio of observed and field-expected citations rate using different granularity.

According to the expectations, the correlation between the ECOOM subfields and the WoS subject categories proved very strong $\left(r^{2}=0.971\right)$, while the correlation between the highest (major fields) and lowest hierarchical level (subject categories) was distinctly weaker, but still quite strong $\left(r^{2}=0.844\right)$. This goes with a larger variance and, most notably, the large number of outliers in the bottom diagram might cause problems in practice. The most significant observation is, however, the closeness of the slope to the value 1.0. This means that the choice of the hierarchical level, i.e., the granularity, has practically no "scale-effect" on the relative indicators. In the present study we repeat this exercise in the context of the Characteristic Scores and Scales (CSS), which can be considered a distributional extension of the notion of 'relative indicators'. Also here we applied both the ECOOM subfield level and the original WoS subject categories. Before we present and discuss the result, we briefly introduce the rudiments of the CSS method.
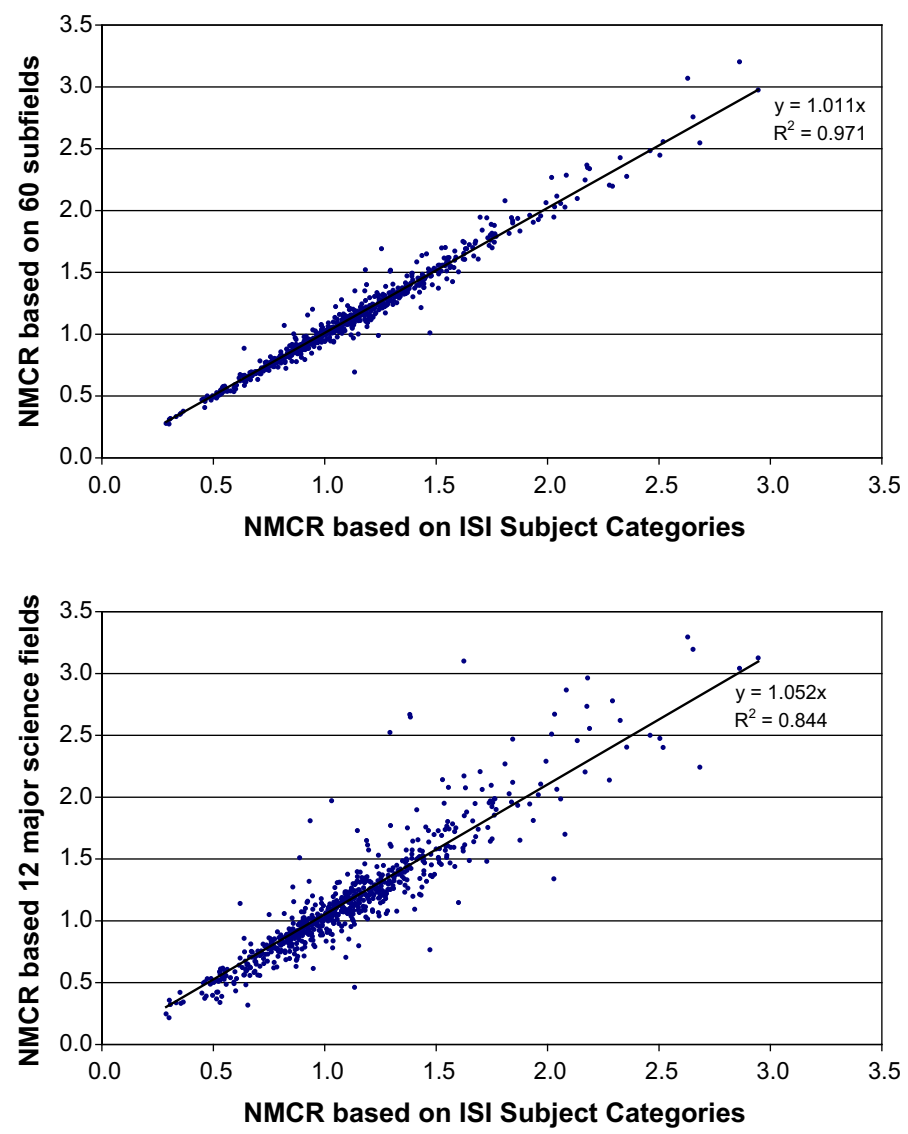

Fig. 1 Plot of subject-normalised citation impact based on ECOOM subfields (top) and major fields (bottom) versus WoS Subject Categories for 676 European universities and research institutions according to Glänzel et al. (2009). (Data sourced from Clarivate Analytics Web of Science Core Collection) 


\section{The granularity of CSS: methods and results}

\section{On the granularity of indicators at the meso level}

As we have mentioned in the previous section, CSS can be considered a non-linear (distributional) extension of field-standardised citation rates. The basic idea is to replace citation indicators by citation profiles that are based on a given number of "performance" classes. The idea behind the method was two-fold, firstly, to favour a parameter-free solution for the assessment, that should be insensitive to outliers, subject-specific peculiarities, publication time and citation window, and provides unique assignment of publications to classes, and, secondly, to overcome the traditional linear projection of a multifaceted, multi-dimensional reality. The "reduction" of the original citation distribution to performance classes as proposed by Glänzel and Schubert (1988) seemed to be the ideal way to achieve this objective. Since then a number of studies has analysed the properties of CSS and confirmed the robustness of the method. An analysis of the granularity has, however, not yet been conducted. However, as CSS has recently been applied also to lower levels of aggregation, notably to institutions, journals and individuals, this might be critical because journals, research teams, and individual scientists might have specific publication profiles that are not sufficiently or adequately covered at the level of standard disciplines. Before we analyse the effect of the choice of the hierarchical level of subject assignment for the calculation of CSS classes, we briefly recall their definition and basic properties.

In a nutshell, characteristic scores are obtained by iteratively calculating the mean value of a citation distribution and subsequently truncating this distribution by removing all papers with less citations than the conditional mean. The number of profile classes used for the evaluation is the only "arbitrary" number that needs to be defined in the beginning. Usually four classes are sufficient. In order to obtain fours classes, the process is stopped after three iterations. The four classes stand for 'poorly cited' (Class 1), 'fairly cited' (Class 2), 'remarkably cited' (Class 3) and 'outstandingly cited' (Class 4) papers. Papers in class 3 and 4 can be considered highly cited. Although the method is based on mean values, the huge number of publications underlying the base-line guarantees that the influence of outliers remains marginal. A further issue in comparative assessment at practically any level of aggregation arises from the peculiarities of scholars' communication behaviour in their research domains and disciplines. This results in different disciplinary standards of publication activity and citation impact. In order to be able to apply the method to the assessment of national research output and to multidisciplinary universities, one has to apply kind of "fractional" normalisation, when calculating the threshold values for class assignment. The detailed description of the procedure and its mathematical background is documented in previous studies by the authors (e.g., Glänzel et al. 2014).

Just as all citation indicators, the CSS scores, too, are strongly dependent of the subject matter, but the distribution of papers over the citation classes defined as the intervals between these scores proved largely independent of the subject, publication year and citation window (Glänzel 2007; Glänzel et al. 2014). Although CSS classes are not directly linked to percentiles, notwithstanding that, the baseline derived from the complete population provides a distribution of papers over classes of about $70 \%$ (Class 1), 21\% (Class 2), 6-7\% (Class 3) and 2-3\% (Class 4), independently of publication year and citation window. Also Albarrán and Ruiz-Castillo (2011) found a similar 70-21-9 rule when they combined the two upper performance classes for papers published between 1998 and 2002 
and using a 5-year citation window. This robustness has made the method attractive as universal bibliometric profiling tool.

Before we further elaborate the CSS scheme for studying the effect of granularity, we go briefly back to a more general question. The research profiles of the units of macro- and meso-level analysis reflect multi-disciplinary or at least activities in broader subjects. The effect of specialisation, even within subject areas that are, otherwise, relevant for the units under study, becomes more pronounced at lower levels of aggregation and most notably at the micro level. The specialisation of individual scientist as reflected by their publication profiles can often hardly be captured by pre-defined disciplines or subject categories. The same applies to research topics and sub-disciplines. Scientometrics as a topic or discipline might serve just as an example: The discipline is, on one hand, a highly specialised subdiscipline within information \& library science, and, being a truly inter-disciplinary field, is, on the other hand, related to many other fields as well and its metrics thus need to be compared with various different subject standards. The relationship with other disciplines is established cognitively by the actual application and practically through the journal assignment to the relevant subject categories. However, none of most subjects outside information science is representatively covered by scientometrics. The topic standard may also be distinctly different from that of its mother discipline meaning that gauging literature in a sub-discipline against the main-discipline standard would result in a distorted picture. In the case of scientometrics, actually a higher standard has to be assumed in order to obtain a realistic benchmark (see Glänzel et al. 2018). These are the reasons why the application of subject classification schemes might fail if applied as a basis for the benchmarking specialised topics and the citation impact of individual scientists. The same effect of incomplete or not representative coverage may occur at the meso and micro level, if (specialised) subsets of disciplines do not necessarily represent the standard of the discipline. In both cases the question of the granularity of the underlying classification system emerges. Even, if ambiguity is sufficiently compensated by subject fractionation and weighting (see Glänzel et al. 2014), unlike in the case of higher aggregation levels such as universities and countries (cf. Fig. 1), the granularity might not prove appropriate to provide results of acceptable accuracy if we break down data to the topic, journal or team level. This is the reason why we decided to use an experimental approach to adjusting the "CSS resolution" for subject/topic selection in journal analysis. In order to be able to conduct large-scale analyses, we will study the effect of granularity at the journal level. This allows us to process all data of a complete volume of the WoS data base including the SCIE, SSCI and A\&HCI editions in a systematic way.

For the present study we have chosen the complete 2013 volume of the WoS journal editions. We have only taken so-called citable items, that is, documents of the types: article, letter and review, into account. Again, we have used the two hierarchical levels, the 74 ECOOM disciplines and the roughly $250 \mathrm{WoS}$ subject categories we have used for subject assignment. First, we calculated field standardised citation rate for journals according at both levels of aggregation. Quasi as a by-product, we obtain a subjectinsensitive citation metric that would allow journal ranking across subject fields. Yet, our intention is to overcome linearity in depiction citation impact, therefore we use these measure just as auxiliary tools. Figure 2 shows the plot of subject-normalised mean citation rates for the 3-year citation window 2013-2015 based on ECOOM subfields vs. WoS Subject Categories. Only journals with at least 10 papers in 2013 are plotted and analysed. Since many journals are rather small, i.e., more than half the journals published less than 100 citable papers in 2013, we expected a larger variance and outliers being more frequent than in the case of universities. However, the correlation still proved to be strong 


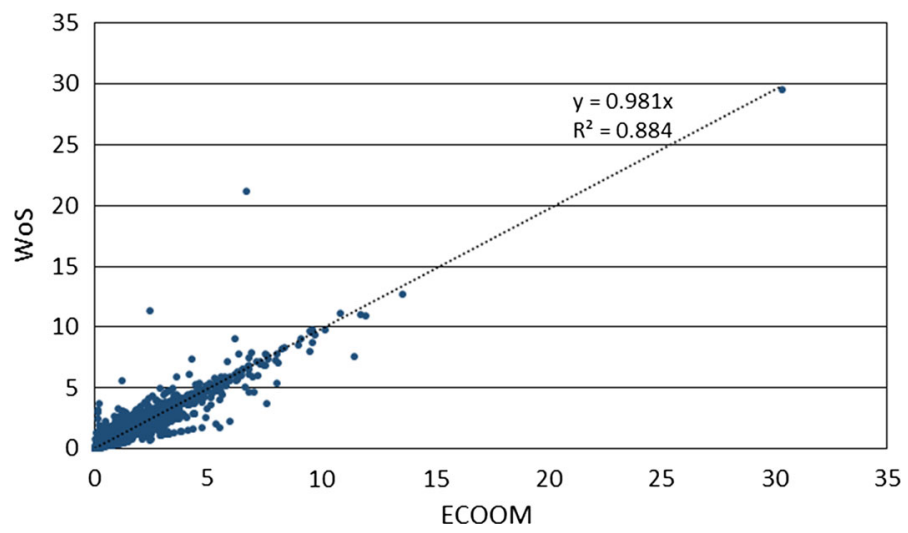

Fig. 2 Plot of subject-normalised mean citation rates based on ECOOM subfields versus WoS Subject Categories for 11,964 journals covered by the WoS database. (Data sourced from Clarivate Analytics Web of Science Core Collection)

and the slope of the linear regression is again close to the value one, substantiating that the level of subject assignment does not affect the scale of the standardised citation index. The presence of an outlier with both values close to 30 did not affect the outcome of the regression analysis.

In the second step we calculated the CSS class distributions for the complete population, that is, for all citable papers indexed and all journals covered in the $2013 \mathrm{WoS}$ volume. The ECCOM classification resulted in the class distribution of $70.0 \%$ (Class 1), $21.4 \%$ (Class 2), $6.2 \%$ (Class 3) and 2.4\% (Class 4), while the corresponding distribution based on WoS categories $69.8,21.4,6.3$ and $2.5 \%$. No statistical test is needed to understand that the deviation of the two distributions from each other is negligible. Nevertheless, we applied a $\chi^{2}$ homogeneity test to test the deviation of class distributions to all individual journals with at least 10 citable papers in 2013. Using the critical value of 7.81 with 3 degrees of freedom and a confidence level of 0.05 , we found that $94.6 \%$ of all journals do not differ significantly if the two levels of subject assignments are applied, while de deviation of $5.4 \%$ of the journals must be considered significant. This is practically in line with the assumed confidence level.

\section{Characteristic scores and scales in journal assessment}

From the historical viewpoint, the use of the CSS in the context of journal assessment was the first application and this was actually the application the method was designed for (see Glänzel and Schubert 1988). Unlike subject assignment or the profiles of individuals and research units or countries, journals do not require multiple assignments of papers. Journals form a true partition of the document space that is covered by a bibliographic database and also of any paper set under study. If papers are to be assigned to journals on the basis where they have been published, assignment is always unique and thus no fractionation is needed. This property essentially simplifies the application of bibliometrics to journal indicators. On the other hand, journal analysis is still an important methodological topic in scientometric research and an indispensable fundament for supplementing bibliographic databases by journal metrics (cf. Journal Citation Reports (JCR), Scimago Journal Ranking (SJR), 
Scopus CiteScore metrics) and as long as journals serve as the basis of subject classification, journal indicators remain essential issues in bibliometric studies.

In the first applications, CSS were applied to chemistry journals to gauge journals Mean Citation Rates against the CSS standards calculated for each subfield of chemistry. At that time the share of uncited paper was still used as a supplementary Class 0 (below the poorly cited articles). Later on, we restrained from this solution because of lacking robustness: The share of uncited papers strongly depends on both the citation windows and the subject matter. The reason for the instability is that this is the only class that is defined on a fixed criterion (i.e., uncitedness), while all other classes are based on variable thresholds that depend on time and research topic. The basic idea at that time was to provide additional information about the journals' position in a discipline beyond the usual ranking exercises. Schubert et al. (1989) already applied CSS to journals, now on the basis of two scores only and with the purpose to gauge national contribution to journal impact but not to compare class distributions by journals. Now we will use CSS class distributions as an alternative to journal ranking. This also demonstrates a new and completely different application context. Although this kind of application does not allow any linear ranking and the interpretation of CCS class distributions is not always straightforward, the added value of information and the more detailed picture of citation impact that we obtain compensates for the lack of simplicity. The surplus of information results from two sources that have been addressed many times in the scientometric literature, first the subject-specific peculiarities of citation impact and, second, the various shapes of citation distributions underlying journal impact. Glänzel and Moed (2002) and Glänzel (2009) have given examples of journals with almost identical Mean Citation Rates but different distribution shapes. We have computed Mean Citation Rates (MCR) and CSS class distribution for all journals in 2013 using a 3-year citation window. We have applied the fractionation process for subjects according to the procedure described in Glänzel et al. (2009) since many journals have multiple subject assignments. Table 1 gives the MCR values of 5 selected journal pairs with almost identical citation impact (MCR) each but distinctly different profile types according to their CSS class distributions. We have chosen journals representing different "standards" to illustrate that this phenomenon might occur in all impact classes ranging from high to low standard.

\section{Granularity at the limits: journal-based benchmarking}

In the previous two sub-sections we have applied bibliometric field-based indicators at two different levels of granularities to the profiling of journal impact. Experience showed, on the one hand, that broad fields, such as the 15 major fields according to the LeuvenBudapest classification scheme or the 22 science areas of Clarivate Analytics Essential Science Indicators (ESI) are less suited to serve as the basis of reference standards. In particular, the major fields and broad science domains combine theoretical, experimental, methodological, empirical and applied research in one field. In contrast to this high level of aggregation, Table 2 shows the aggregate impact factors of ten selected subject categories in physics in the 2015 science editions of the Journal Citation Reports. It is clear that there can hardly be found a common reference standard for, e.g., condensed matters (CMPh) and mathematical physics. The barrier for applying broad fields is therefore two-fold, we are faced with cognitive reasons (comparability-why compare mathematical physics or acoustics with $\mathrm{CMPh}$ ?) as well as different metric standards (commensurability) if we choose to broad subject fields for subject normalisation. 
Table 1 Examples of selected journal pairs with almost identical mean citation rates (MCR) but different CSS profiles in 2013 with 3-year citation window, based on ECOOM subfields (1st row) and WoS Categories (2nd row). $\chi^{2}$-values indicate difference between $1 \mathrm{st}$ and $2 \mathrm{nd}$ row with $3 \mathrm{df}$ 's. (Data sourced from Clarivate Analytics Web of Science Core Collection)

\begin{tabular}{|c|c|c|c|c|c|}
\hline Journal title & Statistics & $\begin{array}{l}\text { Class } 1 \\
(\%)\end{array}$ & $\begin{array}{l}\text { Class } 2 \\
(\%)\end{array}$ & $\begin{array}{l}\text { Class } 3 \\
(\%)\end{array}$ & $\begin{array}{l}\text { Class } 4 \\
(\%)\end{array}$ \\
\hline \multirow[t]{2}{*}{ Advanced Energy Materials $(n=157)$} & $\mathrm{MCR}=31.92$ & 4.5 & 29.3 & 33.1 & 33.1 \\
\hline & $\chi^{2}=1.59$ & 7.6 & 29.9 & 32.5 & 29.9 \\
\hline \multirow[t]{2}{*}{ Molecular Biology and Evolution $(n=238)$} & $\mathrm{MCR}=31.71$ & 42.0 & 42.0 & 13.0 & 2.9 \\
\hline & $\chi^{2}=0.00$ & 42.0 & 42.0 & 13.0 & 2.9 \\
\hline \multirow[t]{2}{*}{$\operatorname{AJRCCM}(n=460)$} & $\mathrm{MRC}=15.84$ & 42.4 & 20.4 & 23.0 & 14.1 \\
\hline & $\chi^{2}=3.94$ & 44.8 & 23.3 & 21.5 & 10.4 \\
\hline \multirow[t]{2}{*}{ BMC Medicine $(n=267)$} & $\mathrm{MCR}=15.75$ & 28.1 & 52.8 & 17.6 & 1.5 \\
\hline & $\chi^{2}=7.66$ & 28.1 & 61.0 & 10.1 & 0.7 \\
\hline \multirow[t]{2}{*}{ Scientific Reports $(n=2455)$} & $\mathrm{MCR}=11.16$ & 61.1 & 31.7 & 5.8 & 1.4 \\
\hline & $\chi^{2}=0.00$ & 61.1 & 31.7 & 5.8 & 1.4 \\
\hline \multirow[t]{2}{*}{ Nutrition Reviews ( $n=83$ ) } & $\mathrm{MCR}=11.16$ & 21.7 & 38.6 & 27.7 & 12.0 \\
\hline & $\chi^{2}=6.15$ & 38.6 & 26.5 & 22.9 & 12.0 \\
\hline \multirow[t]{2}{*}{ Journal of Lightwave Technology $(n=578)$} & $\mathrm{MCR}=5.60$ & 51.6 & 29.4 & 13.8 & 5.2 \\
\hline & $\chi^{2}=2.53$ & 51.6 & 32.5 & 11.4 & 4.5 \\
\hline \multirow[t]{2}{*}{ Clinica Chimica Acta $(n=364)$} & $\mathrm{MCR}=5.60$ & 72.0 & 22.3 & 4.4 & 1.4 \\
\hline & $\chi^{2}=16.08$ & 58.8 & 30.8 & 6.3 & 4.1 \\
\hline \multirow{2}{*}{$\begin{array}{l}\text { International Journal of Number Theory } \\
(n=120)\end{array}$} & $\mathrm{MCR}=0.90$ & 74.2 & 20.8 & 4.2 & 0.8 \\
\hline & $\chi^{2}=0.00$ & 74.2 & 20.8 & 4.2 & 0.8 \\
\hline \multirow{2}{*}{$\begin{array}{l}\text { Advances in Materials Science and } \\
\text { Engineering }(n=248)\end{array}$} & $\mathrm{MCR}=0.90$ & 97.2 & 2.8 & 0.0 & 0.0 \\
\hline & $\chi^{2}=1.63$ & 98.8 & 1.2 & 0.0 & 0.0 \\
\hline
\end{tabular}

Table 2 Aggregate Impact Factors (AIF) of ten subject categories in Physics according to the 2015 Sciences Edition of the JCR. (Data sourced from Clarivate Analytics Journal Citation Report)

\begin{tabular}{lc}
\hline Subject category & AIF \\
\hline Physics, condensed matter & 4.023 \\
Physics, particles and fields & 3.880 \\
Physics, applied & 3.261 \\
Physics, atomic, molecular and chemical & 3.028 \\
Physics, nuclear & 2.788 \\
Optics & 2.220 \\
Crystallography & 2.219 \\
Physics, fluids and plasmas & 2.094 \\
Acoustics & 1.853 \\
Physics, mathematical & 1.802 \\
\hline
\end{tabular}

On the other hand, if the narrower fields or disciplines, such as the WoS subject categories are chosen, the already discussed issues of fuzziness and ambiguity challenge both methodology and its implementation. Multiple journals assignment to subjects becomes more frequent and results in sometimes exuberant fractionation and weighting 
that is needed to calculate and apply reference standards and CSS scores. Without the application of such procedure the choice of an appropriate reference value would be impossible as has been shown using the example of the journal Bioinfomatics that took deviating positions in journal ranking according to the different WoS categories it was assigned to (cf. Glänzel 2011). An arbitrary restriction to single assignment at this level would, however, hardly be justified from the cognitive viewpoint nor provide accurate reference standards for any indicators to be built on the assignment.

Recalling the results by Zitt et al. (2005), who referred to "the coexistence of various perspectives each having their own form of legitimacy", we see a further limitation to increasing the resolution, even if we respect the legitimacy of different granularities. As we already have confirmed this notion in the context of relative mean citation rates in our section on general methodological issues, subject- and journal-based reference standards do reflect different perspectives. We have calculated the journal based relative citation rate (RCR) as well its sub-field based pendent (NMCR). The different concepts underlying these structures becomes apparent from the comparison of the national indicators that are given in Table 3. For this table we have compiled a list of the 25 most active countries.

All citation indicators have been calculated on the basis of the 3-year citation window 2013-2015. The low NMCR values of India, Iran, Turkey, Russia and Brazil are contrasted by their moderate or even high journal-based counterparts (RCR) indicating that these countries are publishing in rather low-impact journals. The direct comparison of the two underlying expected citation rates make this even more visible: For Iran, Turkey and Russia, the ratio of the journal and discipline based expectation ranges around 0.6. The comparison of the corresponding CSS classes confirms and deepens these observations. The first observation here concerns the baseline itself. The distribution of papers over CSS classes in the complete population changes towards a lower share of poorly cited and a consequently higher share of fairly and highly cited papers. This has also a strong effect mainly on countries with low impact: In countries with low citation impact, the share of poorly cited papers calculated from journal model is usually greater that the corresponding field-based share. Countries with generally high citation impact are not affected by this trend, on the contrary, as can been seen for Denmark, Switzerland, the Netherlands or Belgium. For these countries we also observe somewhat higher field-based than journalbased expectations. The ratio here amounts to about 1.3. In both extreme cases, we have looked at, we see the effect of national "publication strategies", namely, in favour of publishing in low or high impact journals with respect to their corresponding subject standards. In this context we would like to recall the effect of choosing publication channels and fora already mentioned in the introduction.

However, apart from these perspectives a real barrier emerges, namely that given by the rules of mathematical statistics - at least in the context of CSS. And in this context, we have to stress that the limitation applies to the population, not to the size of the sample units under study. The commonly accepted minimum for the application of mathematical statistics to mean-value and quantile based statistics lies around 30-40 items (cf. Vincze 1974). WoS subject categories meet this criterion but about one quarter of all journals have less than 30 citable paper per year. For the calculation of reference standards for mean values and shares this is still acceptable. Now, analogously, the use of journals instead of subject categories or disciplines could be an interesting extension to journal-based expectation also in the framework of CSS. In other words, journal-based characteristic scores and scales could supplement the field based CSS giving another perspective to citation-class profiling. However, here the distribution based approached is stretched to its limits. For instance, if we assume a size of 40 items in the reference journal, the share of 
Table 3 Discipline (NMCR) and journal based (RCR) relative citation rates and CSS classes based on a 3 -year citation window for the 25 most active countries in 2013. (Data sourced from Clarivate Analytics Web of Science Core Collection)

\begin{tabular}{|c|c|c|c|c|c|c|c|c|c|c|c|}
\hline \multirow[t]{2}{*}{ Country } & \multirow[t]{2}{*}{ Papers } & \multicolumn{5}{|c|}{ Discipline based } & \multicolumn{5}{|c|}{ Journal based } \\
\hline & & NMCR & $\begin{array}{l}\mathrm{C} 1 \\
(\%)\end{array}$ & $\begin{array}{l}\mathrm{C} 2 \\
(\%)\end{array}$ & $\begin{array}{l}\text { C3 } \\
(\%)\end{array}$ & $\begin{array}{l}\mathrm{C} 4 \\
(\%)\end{array}$ & RCR & $\begin{array}{l}\mathrm{C} 1 \\
(\%)\end{array}$ & $\begin{array}{l}\mathrm{C} 2 \\
(\%)\end{array}$ & $\begin{array}{l}\text { C3 } \\
(\%)\end{array}$ & $\begin{array}{l}\mathrm{C} 4 \\
(\%)\end{array}$ \\
\hline Australia & 54,205 & 1.39 & 60.6 & 26.4 & 8.9 & 4.2 & 1.18 & 59.6 & 25.3 & 9.1 & 6.0 \\
\hline Austria & 14,094 & 1.43 & 59.9 & 26.7 & 9.2 & 4.3 & 1.20 & 59.2 & 25.1 & 9.2 & 6.6 \\
\hline Belgium & 21,039 & 1.55 & 58.3 & 27.1 & 9.7 & 4.9 & 1.25 & 58.8 & 25.2 & 9.3 & 6.7 \\
\hline Brazil & 39,499 & 0.76 & 78.8 & 16.1 & 3.6 & 1.4 & 0.98 & 66.9 & 22.1 & 6.7 & 4.2 \\
\hline Canada & 64,137 & 1.37 & 62.2 & 25.4 & 8.4 & 3.9 & 1.14 & 62.3 & 23.8 & 8.3 & 5.6 \\
\hline China & 218,125 & 1.01 & 69.6 & 21.3 & 6.4 & 2.7 & 1.11 & 62.7 & 24.4 & 8.4 & 4.5 \\
\hline Denmark & 15,625 & 1.64 & 55.2 & 28.8 & 10.6 & 5.4 & 1.28 & 57.5 & 26.5 & 9.5 & 6.5 \\
\hline France & 72,824 & 1.33 & 63.1 & 25.1 & 8.0 & 3.8 & 1.11 & 63.0 & 23.4 & 7.9 & 5.7 \\
\hline Germany & 102,566 & 1.37 & 61.2 & 26.2 & 8.6 & 4.0 & 1.14 & 60.8 & 24.6 & 8.8 & 5.8 \\
\hline India & 54,083 & 0.77 & 76.2 & 18.0 & 4.4 & 1.5 & 1.03 & 65.4 & 22.7 & 7.7 & 4.2 \\
\hline Iran & 26,665 & 0.74 & 77.1 & 16.7 & 4.5 & 1.7 & 1.16 & 62.3 & 23.6 & 8.6 & 5.5 \\
\hline Israel & 13,169 & 1.36 & 65.6 & 23.8 & 7.2 & 3.4 & 1.08 & 66.7 & 21.6 & 7.0 & 4.6 \\
\hline Italy & 64,334 & 1.26 & 62.4 & 26.0 & 8.3 & 3.4 & 1.16 & 59.9 & 25.2 & 8.9 & 5.9 \\
\hline Japan & 79,048 & 0.95 & 73.6 & 19.5 & 4.9 & 2.0 & 0.95 & 68.6 & 21.3 & 6.6 & 3.5 \\
\hline Netherlands & 38,227 & 1.61 & 55.6 & 28.7 & 10.5 & 5.2 & 1.22 & 58.8 & 25.6 & 9.3 & 6.3 \\
\hline Poland & 24,081 & 0.83 & 76.2 & 17.7 & 4.2 & 1.9 & 1.08 & 63.6 & 23.2 & 8.1 & 5.1 \\
\hline Russia & 29,447 & 0.61 & 84.4 & 11.6 & 2.8 & 1.2 & 1.05 & 66.8 & 21.4 & 7.4 & 4.4 \\
\hline Korea & 50,720 & 0.95 & 72.8 & 19.8 & 5.4 & 2.0 & 0.99 & 66.3 & 22.7 & 7.3 & 3.7 \\
\hline Spain & 56,839 & 1.25 & 64.8 & 24.4 & 7.5 & 3.3 & 1.11 & 63.1 & 23.4 & 7.9 & 5.6 \\
\hline Sweden & 24,719 & 1.47 & 59.5 & 27.2 & 9.1 & 4.2 & 1.20 & 60.3 & 25.0 & 9.0 & 5.7 \\
\hline Switzerland & 27,604 & 1.70 & 54.4 & 28.6 & 11.4 & 5.6 & 1.25 & 57.8 & 25.6 & 9.9 & 6.7 \\
\hline Taiwan & 27,709 & 0.93 & 73.0 & 20.0 & 5.2 & 1.9 & 0.93 & 69.0 & 21.5 & 6.3 & 3.2 \\
\hline Turkey & 27,276 & 0.64 & 81.7 & 13.8 & 3.3 & 1.2 & 1.04 & 66.4 & 21.9 & 7.1 & 4.6 \\
\hline UK & 112,662 & 1.45 & 60.1 & 26.4 & 9.1 & 4.4 & 1.14 & 61.6 & 24.1 & 8.5 & 5.8 \\
\hline USA & 386,556 & 1.38 & 60.7 & 26.2 & 9.0 & 4.1 & 1.07 & 62.6 & 23.7 & 8.3 & 5.4 \\
\hline Total & $1,464,089$ & 1.00 & 70.0 & 21.4 & 6.2 & 2.4 & 1.00 & 65.0 & 22.6 & 7.5 & 4.9 \\
\hline
\end{tabular}

Class 4 items of $2.5 \%$ of the reference distribution would correspond to exactly 1 item. This might, however, challenge the robustness of the method. For this reason, we do recommend a selective extension of CSS to journal-based profile classes.

\section{New aspects of granularity: individual topics as reference baseline}

Journal- and subject-related reference indicators provide efficient standards as long as the documents sets under study generally fits into the framework structure of pre-defined subject classification and journal coverage. In other words, the document sets must form representative parts of the underlying journal and subject structure. Otherwise, the choice 
of granularity, that is, the choice of subject resolution might result in biased indicators. This applies to all indicators including characteristic scores and scales. In order to illustrate this, we will give some examples, but first we would like to stress that the above-mentioned required representativity of subjects and journals is usually guaranteed at the macro and meso level, if research performance of countries or research institution is analysed for all fields, even if those have specific profiles with preferences according to the typical paradigmatic patterns such as life sciences, technical sciences, social sciences, etc. as being predominant. The different citations standards of the individual profiles can readily be compensation by the choice of appropriate indicators (e.g. Glänzel et al. 2009). The situation completely changes, if the document sets under study do not form a representative selection of the underlying subject structure as often observed at the micro level or in the case of interdisciplinary research fields. Individual scientists or teams are usually conducting research and publishing their results in specific topics that are often not in line with the standards of the underlying fields. The reason is very simple: if scientists are, for instance, publishing in topics that are more cited that the corresponding subject standard, a bias towards higher relative citation indicator values might be obtained for an unproportionally large part of scientists. This has been shown for the case of scientometrics (cf. Thijs et al. 2017).

Similarly, for domain studies in interdisciplinary and emerging research fields, predefined subject scheme and journal standards may not provide an adequate baseline structure. Domain studies of nano-science and -technology, stem-cell research and biotechnology (Glänzel et al. 2003, 2004; Glänzel and Zhou 2011) have illustrated that the world total of relative indicators may distinctly differ from the neutral value 1.0, as it should be by definition, if the domain standard represents a higher or lower level than that of the journals and subjects to which the documents are otherwise assigned. This effect cannot be overcome by using any particular choice of granularity regarding the underlying journal and subject structure. Regarding the mutual internal comparison, this is practically not problematic, however, the fact that a disproportionately large share of units or individuals achieves high/low scores and different resolutions may provide different reference values is somewhat bewildering. In order to resolve this issue, an approach beyond the particular choice of subject resolutions must be sought. As all bibliometric methods, characteristic scores and scales are affected by the above-mentioned phenomenon as well. In particular, a practical solution will bring us back to the roots: CSS was originally developed for journal evaluation in selected disciplines and has later been extended to the multi-disciplinary evaluation of national and institutional evaluation. In the case of domain studies, including studies of interdisciplinary and emerging topics, and the citation analysis of individuals and research team, the topic standard of the corresponding domain or the publication output of individuals and teams can provide the baseline distribution for the CSS scores and classes. In order to illustrate the bias caused by using standard subjects based on different granulation, we use the topic "scientometrics" defined on the basis of a combination of cores journals and a number of search strings (cf. Thijs et al. 2017). We have collected all journal papers of document type article, letter and review indexed in Clarivate Analytics WoS published in 2011 and 2012. For comparison, we have used the "mother discipline" information \& library science as defined by the corresponding WoS subject category. Furthermore, we have defined three reference standards, the discipline based resolution of the complete WoS, the subject category information \& library science (LIS) and the topic scientometrics (SCIM). In contrast to the expectations, we do not obtain a clear and strictly descending subset structure (WoS $\supseteq$ LIS $\supseteq$ SCIM) since many SCIM papers are published in multidisciplinary or specialised medical, physics and chemistry 
journals, which are not assigned to the LIS category. As a result, the size of the SCIM set amounts to almost $50 \%$ of that of the LIS category. Citations have been counted in a 3-year citation window each (2011-2013 and 2012-2014, respectively). SCIM papers exhibit a higher citation impact than the LIS category. The series of SCIM $B_{i}$ scores is almost twice as high as the corresponding LIS values (see Table 4). Nevertheless, the citation-class sizes almost coincide and follow the expected series $70 \%$ (Class 1), 21\% (Class 2), 6-7\% (Class 3 ) and $2-3 \%$ (Class 4).

In a second step, we have calculated the distribution of the LIS and SCIM papers over the four CSS classes according to different baseline standards. For LIS we have used the WoS standard (LIS| $\left.\right|_{\text {WoS }}$ ) according using the procedure as used in multidisciplinary context and described above and in previous papers (Glänzel et al. 2014). We have applied the same procedure to the SCIM set as well (SCIM| $\left.\right|_{\text {WoS }}$ ) and, in addition, we have also used the LIS standard $\left(\left.\mathrm{SCIM}\right|_{\text {LIS }}\right)$. The results given in Table 5 are interesting in several respects. On one hand, they illustrate the impact bias for both topics, the LIS category and scientometrics since in both cases the $\left.{ }^{*}\right|_{\text {WoS }}$ values reflect lower Class1 shares along with higher Class2-Class4 shares each (with respect to the 70-21-6.6-2.5 rule). Furthermore, the

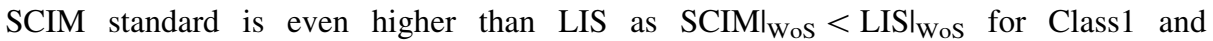
$\left.S C I M\right|_{W o S}>$ LISI $_{\text {WoS }}$ for the higher classes. On the other hand, we observe SCIM| $\left.\right|_{\text {LIS- }}$ $<\left.\mathrm{SCIM}\right|_{\mathrm{WoS}}$ for Class1 and SCIM| $\left.\right|_{\text {LIS }}>\left.\mathrm{SCIM}\right|_{\mathrm{WoS}}$ for the higher classes although the first two rows of Table 5 would suggest the opposite case. Yet we mentioned that there is not strict relationship of subsets among topics and disciplines, that is, in topological parlance, the joint set of topics and subject categories do not generate a filter. In particular, we have SCIM $\&$ LIS nor does the SCIM delineation match the WoS subject (quasi)partition, while LIS does. Since many SCIM papers have been published in journals outside the LIS category, the observed trend is explicable. This example also implies that granularity solutions for the application of CSS do not necessarily require (partially) ordered subset structures.

After the discussion of these general patterns, we will have a look at the applications in the framework of evaluation exercises. Without discussing questions of statistical reliability and inference, we can conclude that the general effect shown in Tables 4 and 5 can also be found in the national data (Table 6). Nevertheless, we can observe several extreme deviations from the general trend, most notably in Belgium, the Netherlands and partially

Table 4 Characteristic scores and citation classes for the LIS category and scientometrics in 2011-2012 based on 3-year citation windows. (Data sourced from Clarivate Analytics Web of Science Core Collection)

\begin{tabular}{lllrlllll}
\hline Topic & Papers & B1 & \multicolumn{1}{c}{ B2 } & \multicolumn{1}{c}{ B3 } & CSS1 (\%) & CSS2 (\%) & CSS3 (\%) & CSS4 (\%) \\
\hline LIS & 6870 & 2.38 & 6.60 & 12.01 & 70.2 & 20.4 & 6.8 & 2.6 \\
SCIM & 3339 & 4.39 & 11.31 & 21.85 & 70.5 & 21.0 & 5.9 & 2.6 \\
\hline
\end{tabular}

Table 5 Distribution of the LIS and SCIM papers over CSS classes according to different baseline standards. (Data sourced from Clarivate Web of Science Core Collection)

\begin{tabular}{lllcl}
\hline Topic & Class 1 (\%) & Class 2 (\%) & Class 3 (\%) & Class 4 (\%) \\
\hline LIS| $\left.\right|_{\text {WoS }}$ & 65.0 & 22.0 & 9.1 & 3.8 \\
SCIM| & 59.5 & 24.1 & 10.9 & 5.5 \\
SCIM| & 52.4 & 28.0 & 12.5 & 7.1 \\
\hline
\end{tabular}




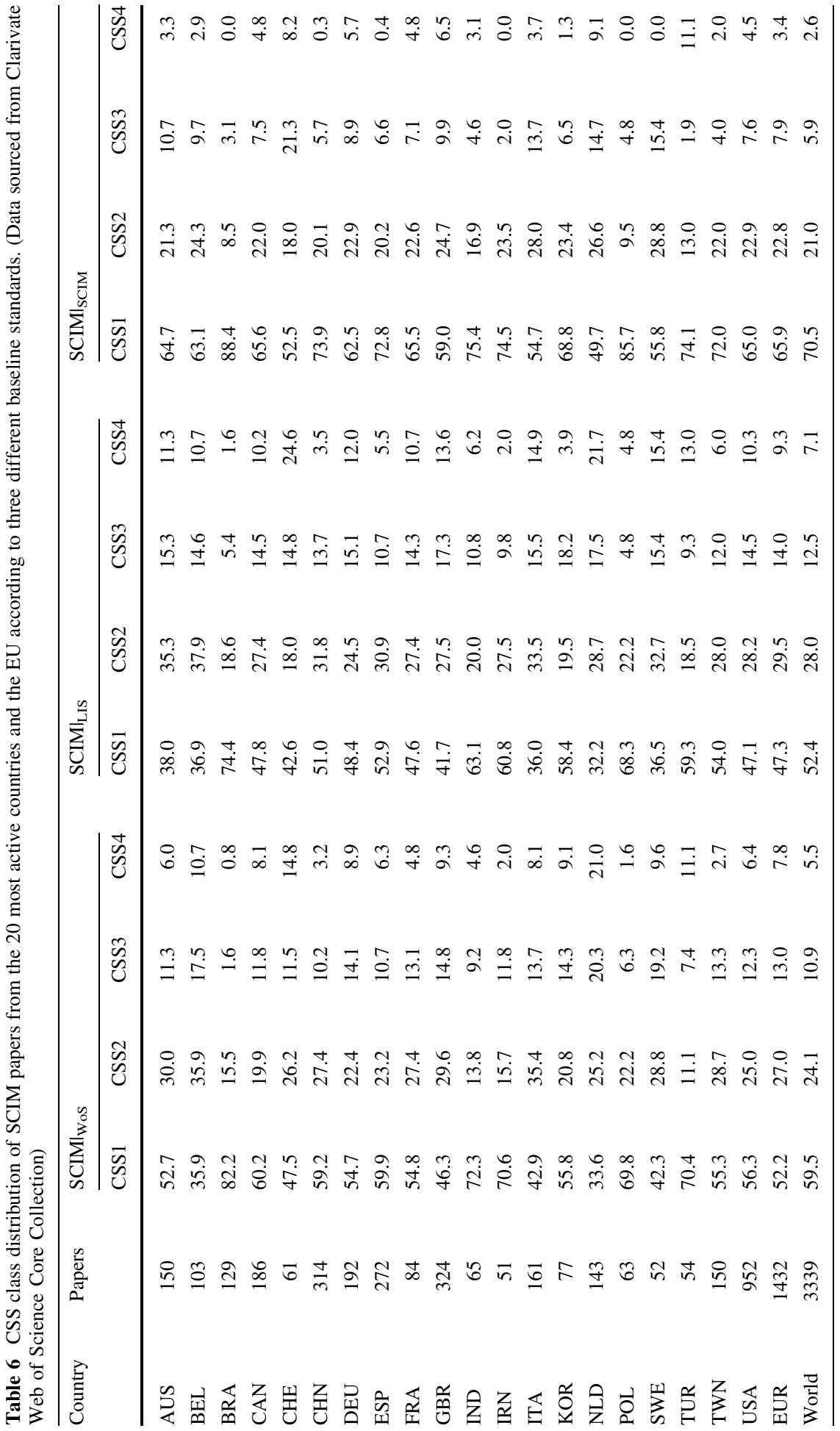


in Korea. Here we find much more advantageous patterns using the discipline and subject standard compared to the own topic standard. The loss of highly cited papers after subject re-assignment is enormous. There is practically no opposite case. Only the extremely polarised case of Turkey with large share of both poorly cited and highly cited papers is still worth mentioning. This trend is nearly independent of the chosen baseline. Once again, we would to stress that the SCIM set does not form a subset of the LIS category. We understand that the possibility of selecting different reference standards for bibliometric indicators impedes comprehensibility and interpretability of results, but their demonstration and discussion in nonetheless necessary to understand the mechanisms underlying scholarly communication patterns.

\section{Conclusions}

We have calculated characteristic scores and scales for journals covered by the Web of Science Core Collection at two levels of granularity, 74 disciplines and the WoS Subject Categories. The results of the overall comparison did not show any substantial deviations that would tell against the robustness of the method. The comparison of CSS and mean citation rates have again substantiated that citation impact is not one-dimensional and should not be mapped by linear functions. Journals may have very similar mean citation rates (impact factors, or more generally, impact measures), while their CSS class distributions distinctly differs, independently of the underlying granularity. Our findings are partially presented on a limited selection of journals. In an extended later version of this study we intend to present data on more journals in a supplementary online material according to the corresponding journals publication policies.

Unlike traditional mean-value or quantile based bibliometric indicators CSS needs somewhat larger reference sets to obtain robust reference distributions. The reason is the higher resolution of the underlying distribution based reference classes. According to the experience made in this study, subject categories already meet this requirement, journals are less suited to build the reference classes for characteristic scores and scales.

A further extension of the concept of granularity is necessary whenever interdisciplinary or otherwise specialised datasets are studied. The application of standard subject- or journal-based baselines may result in biases, which do not produce invalid comparison or evaluation, but may somewhat distort the comprehensibility and interpretability of indicators, notably if studies from different fields and contexts are bundled and used as parts of more comprehensive portfolios. In such cases, refraining from universal standards in favour of individual baselines would be the necessary consequence.

Summarising, the proper choice of granularity has therefore some particular limits, both the heterogeneity of too broad subject fields and an increased resolution towards too narrow topics could affect the validity of results. And sometimes the is no common measure for all cases. One size does definitely not fit all. As so often, when turning theory to practice, the optimum is somewhere in between.

Acknowledgements The present study is an extended version of an article presented at the 16th International Conference on Scientometrics and Informetrics, Wuhan (China), 16-20 October 2017 (Glänzel and Thijs 2017). In particular, the paper has been extended by adding a new option based on new data and results, which are presented in a new section on topic-based granularity. Figure 1 has been reproduced from Glänzel et al. (2009) with permission of the publisher. Table 1 presents data on subfield-based journal indicators shared with figures of Table 9 in Glänzel et al. (2018). 


\section{References}

Adams, J., Gurney, K., \& Jackson, L. (2008). Calibrating the zoom-A test of Zitt's hypothesis. Scientometrics, 75(1), 81-95.

Adams, J., \& Testa, J. (2011). Thomson Reuters book citation index. In E. Noyons, P. Ngulube, J. Leta (Eds.), The 13th conference of the international society for scientometrics and informetrics (Vol. I, pp. 13-18). Durban, South Africa: ISSI, Leiden University and the University of Zululand.

Albarrán, P., \& Ruiz-Castillo, J. (2011). References made and citations received by scientific articles. JASIST, 62(1), 40-49.

Bookstein, A. (1997). Informetric distributions. 3. Ambiguity and randomness. Journal of the American Society for Information Science, 48(1), 2-10.

Glänzel, W. (2007). Characteristic scores and scales. A bibliometric analysis of subject characteristics based on long-term citation observation. Journal of Informetrics, 1(1), 92-102.

Glänzel, W. (2009). The multi-dimensionality of journal impact. Scientometrics, 78(2), 355-374.

Glänzel, W. (2011). The application of characteristic scores and scales to the evaluation and ranking of scientific journals. Journal of Information Science, 37(1), 40-48.

Glänzel, W., Meyer, M., Schlemmer, B., du Plessis, M., Thijs, B., Magerman, T., Debackere, K., Veugelers, R. (2003), Nanotechnology-Analysis of an emerging domain of scientific and technologic endeavour. Accessible via: https://www.ecoom.be/sites/ecoom.be/files/downloads/nanotech_domain_study.pdf.

Glänzel, W., \& Moed, H. F. (2002). Journal impact measures in bibliometric research. Scientometrics, 53(2), $171-193$.

Glänzel, W., \& Schubert, A. (1988). Characteristic scores and scales in assessing citation impact. Journal of Information Science, 14(2), 123-127.

Glänzel, W., \& Schubert, A. (2003). A new classification scheme of science fields and subfields designed for scientometric evaluation purposes. Scientometrics, 56(3), 357-367.

Glänzel, W., Schubert, A., Thijs, B., \& Debackere, K. (2009). Subfield-specific normalized relative indicators and a new generation of relational charts: Methodological foundations illustrated on the assessment of institutional research performance. Scientometrics, 78(1), 165-188.

Glänzel, W., \& Thijs, B. (2017). The granularity of disciplinary structures for benchmarking citation impact. The case of CSS profiles. In Proceedings of ISSI 2015-The 15th international conference on scientometrics and informetrics (pp. 1190-1200). Wuhan, China.

Glänzel, W., Thijs, B., \& Debackere, K. (2014). The application of citation-based performance classes to the disciplinary and multidisciplinary assessment in national comparison and institutional research assessment. Scientometrics, 101(2), 939-952.

Glänzel, W., Thijs, B., \& Debackere, K. (2018). Citation classes: A distribution-based approach to profiling citation impact for evaluative purposes. In W. Glänzel et al. (Eds.), Springer handbook of science and technology indicators. Heidelberg: Springer. to be published.

Glänzel, W., Verbeek, A., du Plessis, M., van Looy, B., Magerman, T., Thijs, B., Schlemmer, B., Debackere, K., Veugelers, R. (2004), Stem cells-Analysis of an emerging domain of scientific and technological endeavour. Accessible via: https://www.ecoom.be/sites/ecoom.be/files/downloads/stemcells_domain_ study.pdf.

Glänzel, W., \& Zhou, P. (2011). Publication activity, citation impact and bi-directional links between publications and patents in biotechnology. Scientometrics, 86(2), 505-525.

Lehmann, F. (2013). Realität und imagination. Photographie in W. G. Sebalds Austerlitz und Michelangelo Antonionis blow up. Bamberg: University of Bamberg Press.

Schubert, A., Glänzel, W., \& Braun, T. (1989). Scientometric datafiles. A comprehensive set of indicators on 2649 journals and 96 countries in all major fields and subfields 1981-1985. Scientometrics, 16(1-6), 3-478.

Thijs, B., Debackere, K., \& Glänzel, W. (2017). Improved author profiling through the use of citation classes. Scientometrics, 111(2), 829-839.

Vincze, I. (1974). Mathematical Statistics, 4th edn. University Script, Eötvös University Budapest (in Hungarian).

Zitt, M., Ramanana-Rahary, S., \& Bassecoulard, E. (2005). Relativity of citation performance and excellence measures: From cross-field to cross-scale effects of field-normalization. Scientometrics, 63(2), 373-401. 